\title{
Cone Beam X-Ray Luminescence Tomography Imaging Based on KA-FEM Method for Small Animals
}

\author{
Dongmei Chen, ${ }^{1}$ Fanzhen Meng, ${ }^{2}$ Fengjun $\mathrm{Zhao}^{3}$ and Cao $\mathrm{Xu}^{2}$ \\ ${ }^{1}$ College of Life Information Science and Instrument Engineering, Hangzhou Dianzi University, Hangzhou 310018, China \\ ${ }^{2}$ School of Life Sciences and Technology, Xidian University, Xian 710071, China \\ ${ }^{3}$ School of Information Sciences and Technology, Northwest University, Xian, Shaanxi 710069, China \\ Correspondence should be addressed to Cao Xu; caoxu327@gmail.com
}

Received 21 June 2016; Revised 6 September 2016; Accepted 5 October 2016

Academic Editor: Jinchao Feng

Copyright (C) 2016 Dongmei Chen et al. This is an open access article distributed under the Creative Commons Attribution License, which permits unrestricted use, distribution, and reproduction in any medium, provided the original work is properly cited.

Cone beam X-ray luminescence tomography can realize fast X-ray luminescence tomography imaging with relatively low scanning time compared with narrow beam X-ray luminescence tomography. However, cone beam X-ray luminescence tomography suffers from an ill-posed reconstruction problem. First, the feasibility of experiments with different penetration and multispectra in small animal has been tested using nanophosphor material. Then, the hybrid reconstruction algorithm with KA-FEM method has been applied in cone beam X-ray luminescence tomography for small animals to overcome the ill-posed reconstruction problem, whose advantage and property have been demonstrated in fluorescence tomography imaging. The in vivo mouse experiment proved the feasibility of the proposed method.

\section{Introduction}

X-ray luminescence tomography (XLT) has been put forward as a novel imaging technology for biological imaging application based on X-ray-excitable phosphor nanoparticles [1]. These phosphor nanoparticles can produce visible luminescence light signals irradiated with X-ray which can be measured by charge coupled device (CCD) [1]. The discovery that both X-ray and visible light can propagate through tissue and that the nanophosphors agents can trace specific molecular makes XLT a proper tool for in vivo biomedical imaging. Nowadays, XLT technology has also been extended from narrow beam X-ray [1] to cone beam X-ray excitation [2] and is even applied in endoscopic imaging [3]. Meanwhile, with advanced specific materials for X-ray excitation, it has also been applied in small animal in vivo imaging [4].

The XLT modality has its unique features over other optical imaging methods such as bioluminescence and fluorescence imaging $[5,6]$. It can excite the nanophosphors from different angles and avoid a significant autofluorescence in other optical imaging methods [2]. However, this technology demands long scanning time under X-ray exposure, which limits its development of fast in vivo biology processes [5].
Some research groups have improved the XLT imaging time resolution from different ways. Carpenter et al. proposed a limited-angle X-ray luminescence tomography method to complete reconstruction from limited-angles in narrow beam XLT system [7]. Badea et al. invented a battleship sampling paradigm to mix sampling and reconstruction in narrow beam XLT system [8]. In addition, Chen et al. designed a cone beam XLT imaging system [2] and Chen et al. put forward a reconstruction method with single view data in cone beam XLT system [3]. Even though the imaging time in the reconstruction method with single view data is reported to be less than $30 \mathrm{~s}$ [3], reconstructed result with planar detectors and a single view is generally insufficient for accurate $3 \mathrm{D}$ reconstruction [9]. The inverse problem of reconstruction is an ill-posed problem and can be improved by taking images of the experiment subject from multiple views [10] and using multispectral data [11].

It is reported that $\mathrm{Gd}_{2} \mathrm{O}_{2} \mathrm{~S}$ : $\mathrm{Tb}$ has several peaks in the spectrum excited by $\mathrm{X}$-rays and can be applied in multispectral imaging and reconstruction [12]. The multispectral property of this material has also been reported for its advantage in improving the XLT imaging quality [13]. However, the penetration property of this material under different spectral has 
not been discussed before. In this paper, the spectral property of the material in different tissues has been studied. The feasibility of conducting experiments in small animal has been proved through the preliminary results. The hybrid reconstruction algorithm with Kirchhoff approximation and finite element method (KA-FEM) has been studied in fluorescence tomography to overcome the ill-posedness in reconstruction [14]. Hence, we realize cone beam XLT reconstruction with KA-FEM method and perform experiments on both tissue and in vivo mouse. Our reconstructed results show that the KA-FEM method can be applied in reconstruction to make XLT imaging feasible for small animal imaging.

\section{Method}

In the cone beam XLT system, X-rays are emitted from the X-ray source and travel through the tissues based on Lambert-Beers' law. Once the nanophosphors are irradiated by X-rays, they will emit visible light. The light transport in biological tissues can be accurately modeled by diffusion approximation, owing to the highly scattering and weakly absorbing properties of the soft tissues in the spectral region. The imaging model can be expressed as follows:

$$
\begin{aligned}
& X(\mathbf{r})=X_{0} \exp \left\{-\int_{\mathbf{r}_{0}}^{\mathbf{r}} \mu_{t}(\tau) d \tau\right\}, \\
& S(\mathbf{r})=\varepsilon X(\mathbf{r}) \rho(\mathbf{r}), \\
& -\nabla \cdot[D(\mathbf{r}) \nabla \Phi(\mathbf{r})]+\mu_{a}(\mathbf{r}) \Phi(\mathbf{r})=S(\mathbf{r}) \mathbf{r} \in \Omega,
\end{aligned}
$$

where $X_{0}$ is the $\mathrm{X}$-ray source intensity with the initial position $\mathbf{r}_{0}$ and $\mu_{t}(\tau)$ is the X-ray attenuation coefficient at position $\tau$ that can be computed from X-ray transmission data using an attenuation-based computed tomography (CT) technique. $S(\mathbf{r})$ is the light source. $X(\mathbf{r})$ is the X-ray intensity, and $\rho(\mathbf{r})$ is the nanophosphor density at position $\mathbf{r}$. $\varepsilon$ is the light yield, while $\mathbf{r}$ is the position vector. $\Omega$ is the domain under consideration, and $D(\mathbf{r})=\left(3\left(\mu_{a}(\mathbf{r})+(1-g) \mu_{s}(\mathbf{r})\right)\right)^{-1}$ is the diffusion coefficient with $\mu_{a}(\mathbf{r})$ as the absorption coefficient, $g$ as the anisotropy parameter, and $\mu_{s}(\mathbf{r})$ as the scattering coefficient. $\Phi(\mathbf{r})$ is the photon flux density.

Meanwhile, it is reported that the resolution of the reconstructed results can be significantly improved using data measured at different wavelengths $[15,16]$. With the surface data measured at two or more wavelengths, the significantly different system matrixes can be obtained to enhance the resolution of the problem. The ratio of the energy distribution in every spectrum can be measured and the linear relationship between the measured multispectral data $\Phi^{\text {meas }}$ and the material distribution $q$ can be obtained as follows:

$$
A q=\Phi^{\text {meas }}
$$

where

$$
A=\left[\begin{array}{c}
\omega_{\lambda 1} A_{\lambda 1} \\
\omega_{\lambda 2} A_{\lambda 2} \\
\vdots \\
\omega_{\lambda m} A_{\lambda m}
\end{array}\right],
$$

$$
\begin{aligned}
& \Phi^{\text {meas }}=\left[\begin{array}{c}
\Phi_{\lambda 1} \\
\Phi_{\lambda 2} \\
\vdots \\
\Phi_{\lambda m}
\end{array}\right], \\
& \sum_{\nu=1}^{m} \omega_{\lambda \nu} \approx 1 .
\end{aligned}
$$

$A_{\lambda i}, \Phi_{\lambda i}$, and $\omega_{\lambda i}$ represent the system matrix, the measurable photon density, and the relative fraction at which the wavelength $\lambda i$ contributes in the emission spectrum, respectively. $\omega_{\lambda i}$ is given as follows:

$$
\omega_{\lambda i}=\frac{\int_{\lambda_{i}^{\mathrm{lo}}}^{\lambda_{i \mathrm{p}}^{\mathrm{up}}} \xi(\lambda) d \lambda}{\int_{0}^{\infty} \xi(\lambda) d \lambda},
$$

where $\lambda_{i}^{\text {lo }}$ and $\lambda_{i}^{\text {up }}$ denote the lower and upper limits of the bandpass filter centered on wavelength $\lambda i$, respectively, and $\xi(\lambda)$ is the emission spectrum.

The KA-FEM method is applied to combine the analytical method and numerical method based on finite element method to solve the imaging model and form the system equations [14]. The flow chart of the reconstruction can be divided into the KA module and the FEM module. KA method is utilized to produce the region of interest (ROI) and then FEM is used to reconstruct the final result. In the KA module, the system matrix $A_{\lambda i}$ can be expressed as $G^{\mathrm{KA}}\left(\mathbf{r}_{\mathbf{s}}, \mathbf{r}\right.$, $\left.\lambda_{\mathbf{i}}\right)$ as follows at the corresponding wavelength $\lambda_{\mathbf{i}}[14,17]$ :

$$
\begin{aligned}
& G^{\mathrm{KA}}\left(\mathbf{r}_{\mathbf{s}}, \mathbf{r}, \boldsymbol{\lambda}_{\mathbf{i}}\right)=g\left(\mathbf{r}_{\mathbf{s}}, \mathbf{r}, \boldsymbol{\lambda}_{\mathbf{i}}\right)+\sum_{p=1}^{N}\left[g\left(\mathbf{r}_{\mathbf{p}}, \mathbf{r}, \boldsymbol{\lambda}_{\mathbf{i}}\right)\right. \\
& \left.\quad+2 C_{\mathrm{nd}} D(\lambda i) \frac{\partial g\left(\mathbf{r}_{\mathbf{p}}, \mathbf{r}, \boldsymbol{\lambda}_{\mathbf{i}}\right)}{\partial \mathbf{n}_{\mathbf{p}}}\right] \frac{\partial G^{\mathrm{KA}}\left(\mathbf{r}_{\mathbf{s}}, \mathbf{r}_{\mathbf{p}}, \boldsymbol{\lambda}_{\mathbf{i}}\right)}{\partial \mathbf{n}_{\mathbf{p}}} \\
& \quad \times \Delta S\left(\mathbf{r}_{\mathbf{p}}\right),
\end{aligned}
$$

where $\partial \mathbf{n}_{\mathbf{p}}$ denotes the outward normal vector at surface point $\mathbf{r}_{\mathbf{p}} . \Delta S\left(\mathbf{r}_{\mathbf{p}}\right)$ denotes local planar discrete area on the surface. $g$ denotes Green's function in infinite medium while $G^{\text {KA }}$ denotes Green's function in medium with boundary. The surface values $\partial G^{\mathrm{KA}}\left(\mathbf{r}_{\mathbf{s}}, \mathbf{r}_{\mathbf{p}}, \boldsymbol{\lambda}_{\mathbf{i}}\right) / \partial \mathbf{n}_{\mathbf{p}}$ can be obtained by the method of images [18]:

$$
\begin{aligned}
\frac{\partial G^{\mathrm{KA}}\left(\mathbf{r}_{\mathbf{s}}, \mathbf{r}_{\mathbf{p}}, \lambda_{\mathbf{i}}\right)}{\partial \mathbf{n}_{\mathbf{p}}} \\
=\frac{-\left[g\left(\mathbf{R}, \mathbf{Z}, \lambda_{\mathbf{i}}\right)-g\left(\mathbf{R}, \mathbf{Z}+C_{\mathrm{nd}} D(\lambda i)\right)\right]}{C_{\mathrm{nd}} D(\lambda i)},
\end{aligned}
$$

where $\mathbf{Z}=\left(\mathbf{r}_{\mathbf{s}}-\mathbf{r}_{\mathbf{p}}\right) \cdot\left(-\mathbf{n}_{\mathbf{p}}\right)$ and $\mathbf{R}=\mathbf{Z}-\left(\mathbf{r}_{\mathbf{s}}-\mathbf{r}_{\mathbf{p}}\right)$. The coefficient $C_{\text {nd }}$ takes into account the refractive index mismatch between both media [19]. Then, preliminary reconstructed results can be obtained by $l_{1}$-norm regularization method, while in the 


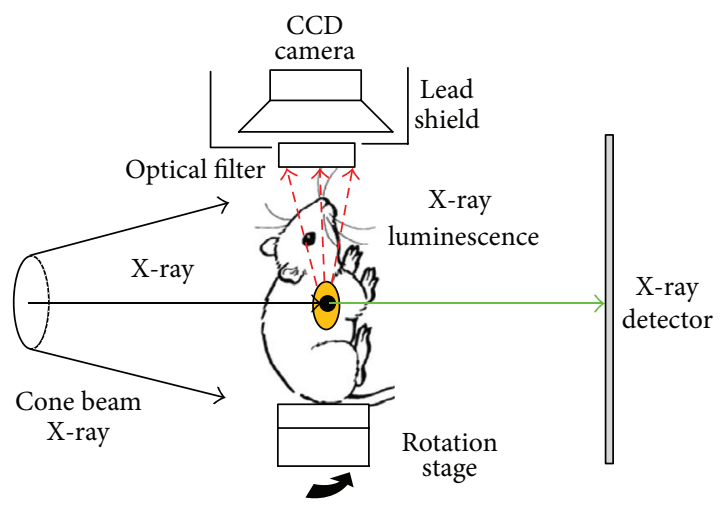

FIGURE 1: Experimental setup for XLT.

FEM module the system matrix $A_{\lambda i}$ can be obtained by transforming the model to its weak form and discretizing the domain with the shape function and is expressed as follows at the corresponding wavelength $\lambda i[2]$ :

$$
A_{\lambda i}=\left(M_{\lambda i}{ }^{-1} F\right) \cdot \varepsilon \cdot X \cdot q
$$

where

$$
\begin{aligned}
M_{i, j}= & \int_{\Omega}\left(D(\lambda i) \nabla \Psi_{i} \cdot \nabla \Psi_{j}+\mu_{a}(\lambda i) \Psi_{i} \cdot \Psi_{j}\right) d r \\
& +\frac{1}{2 \kappa} \int_{\partial \Omega} \Psi_{i} \Psi_{j} d r \\
F_{i, j}= & \int_{\Omega} \Phi(\mathbf{r}) \Psi_{i} \Psi_{j} d r \\
X_{i, j}= & X_{i, j}(\mathbf{r}) .
\end{aligned}
$$

$\varepsilon$ is the light yield and $X_{i, j}(\mathbf{r})$ is the X-ray intensity at each vertex. $\kappa\left(\mathbf{r}, n, n^{\prime}\right)$ is the boundary mismatch factor, which depends on the refractive indices $n$ in $\Omega$ and $n^{\prime}$ in the surrounding medium. $\Psi_{i}$ and $\Psi_{j}$ denote the corresponding elements of the test function. Finally, the reconstructed result can be obtained with conjugate gradient least square (CGLS) method [20].

\section{Experiment and Result}

The equipment used in our experiments consisted of a cone beam X-ray source to excite the phosphors, an electronmultiplying CCD (EMCCD) camera to sample the photon fluence, and an extra CMOS X-ray detector panel to collect the transmitted X-rays. This schematic is shown in Figure 1. In the system, the $\mathrm{CT}$ system contains a microfocus X-ray source (Apogee, Oxford Instruments, UK) and CMOS flatpanel detector (C7921, Hamamatsu, Japan) with pixel size of $50 \mu \mathrm{m}$ covering a $1056 \times 1056$ digital image matrix. The EMCCD camera (PIXIS2048, Princeton Instruments, UK) was mounted at $90^{\circ}$ toward the X-ray axis with a Nikkor 55$\mathrm{mm}$ f/2.8 D lens (Nikon, Melville, New York). To minimize the $\mathrm{X}$-ray ionizing radiation to the EMCCD, a lead shield with depth of $4 \mathrm{~mm}$ was used.
First the depth of penetration and spectral property of our material were investigated. The experiment was performed by using porcine tissues, including liver, kidney, fat, and heart as shown in Figure 2(a). The fresh porcine tissues were frozen at $-20^{\circ} \mathrm{C}$ and then cut into slices of various thicknesses using a microtome with approximately $10 \mathrm{~mm}$ in width and $40 \mathrm{~mm}$ in length. The thicknesses of the porcine tissues were from 2 to $6 \mathrm{~mm}$, with a $2 \mathrm{~mm}$ interval. The material is put into the plastic capillaries with approximately $2 \mathrm{~mm}$ in diameter and $40 \mathrm{~mm}$ in length as shown in Figure 2(a). The distance between the material and the X-ray source was $140 \mathrm{~mm}$ while the distance between the material and EMCCD camera was $285 \mathrm{~mm}$. The capillaries were placed under the porcine tissues of various thicknesses, including 2,4 , and $6 \mathrm{~mm}$. The Xray source was set to $50 \mathrm{kVp}$ voltage and $1 \mathrm{~mA}$ current. The $\mathrm{X}$-ray luminescent photons emitted from the material were acquired by the CCD camera. A $2 \times 2$ binning operation was employed to improve the signal to noise ratio (SNR). During the luminescence signal collection, the exposure time was set to $3 \mathrm{~s}$. The image acquisition system was enclosed in a lighttight environment to avoid the effect of light from outside. The proper optical filters with corresponding wavelengths have been selected based on the optical spectra reported in [12]. The optical signals were imaged at wavelengths of 545, 585 , and $620 \mathrm{~nm}$ with corresponding optical filter manually. The experiment was conducted three times to ensure the accuracy of the measurements.

The luminescent images were obtained as shown in Figure 2(b). The experiment showed that the material can emit visible light signals at wavelengths of 545, 585, and $620 \mathrm{~nm}$ corresponding to the spectrum obtained in [12]. The signals are too weak to detect in our system for the slices with depth of $8 \mathrm{~mm}$. The optical images of the material at different depths and different spectra were acquired and the average of the luminescence flux density in ROI is calculated. Table 1 shows the calculation results of different thickness from different tissues at the wavelength of $620 \mathrm{~nm}$. The color bar in Figure 2(b) and the results of Table 1 show the gray level of the image. Hence, the unit is ADU (Analog to Digital Unit), which can be ignored in both Figure 2(b) and Table 1. Table 1 shows that the optical signal intensity decreased with the increase in depth. Preliminary experimental results indicated that the penetration depth was different in various porcine tissues and that the optical signals for the material at a depth of $6 \mathrm{~mm}$ could be detected for about $3 \mathrm{~g}$ material shown in Figure 2(b). The results prove that this material can be applied in the mouse experiment where the sizes of tissues are relatively small; however, we cannot give the precise measurement including all the tissues such as bones and lung under present conditions. Hence, due to the limitation of the material and system, the radius of the mouse selected in the following experiment is about $20 \mathrm{~mm}$ and the sizes of tissues are smaller than $6 \mathrm{~mm}$, which is in the range of the detectable depth.

Then, an in vivo mouse experiment was carried out in the above imaging system to evaluate the proposed cone beam Xray luminescence computed tomography strategy. To evaluate the performance of the proposed method, we implanted a plastic capillary with a $1 \mathrm{~mm}$ radius and $2 \mathrm{~mm}$ height, which was filled with the $\mathrm{Gd}_{2} \mathrm{O}_{2} \mathrm{~S}$ : $\mathrm{Tb}$ nanophosphor, into the 


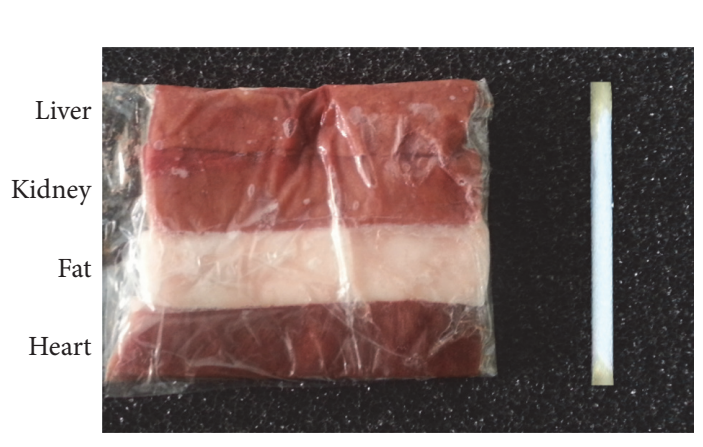

(a)

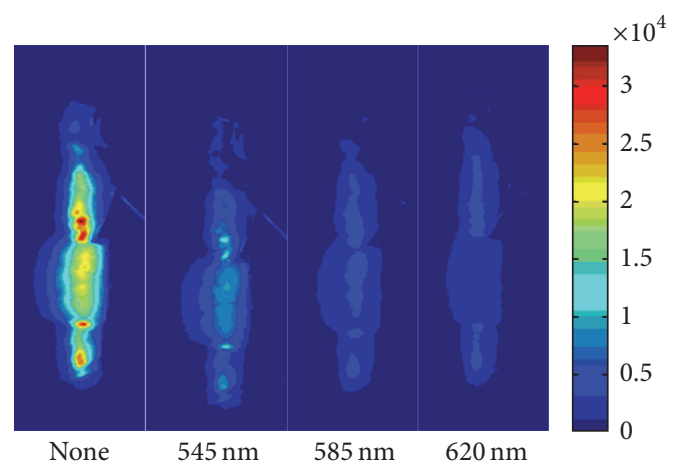

(b)

Figure 2: (a) Slices of different porcine tissues (including liver, kidney, fat, and heart) and the material in the plastic capillaries; (b) slice images under different filters.

TABLE 1: Calculation results of different thicknesses from different tissues at the wavelength of $620 \mathrm{~nm}$.

\begin{tabular}{lccc}
\hline & $2 \mathrm{~mm}$ & $4 \mathrm{~mm}$ & $6 \mathrm{~mm}$ \\
\hline Liver & 252.36 & 18.58 & 6.95 \\
Kidney & 618.96 & 75.77 & 27.84 \\
Fat & 713.06 & 272.33 & 121.79 \\
Heart & 420.17 & 94.15 & 59.83 \\
\hline
\end{tabular}

mouse with the depth of about $10 \mathrm{~mm}$, to simulate tumor applications. The proper optical filters with corresponding wavelengths have been selected the same as in the tissue slices. The distance between the material and the X-ray source was $200 \mathrm{~mm}$ while the distance between the material and EMCCD camera was $270 \mathrm{~mm}$. The micro-CT scanning was also performed $\left(50 \mathrm{kVp}, 1.0 \mathrm{~mA}, 360\right.$ views with $1^{\circ}$ intervals) in the experiment to get the physical structure and the corresponding X-ray attenuation coefficient of the mouse. Then, the X-ray luminescent material was excited by an Xray source from four different directions and the luminescent photons emitted from the phantom were acquired by the CCD camera as shown in Figure 3(a). During the luminescence signal collection, the exposure time was set to $5 \mathrm{~s}$ for each wavelength separately. The optical signals were imaged at wavelengths of 545,585, and $620 \mathrm{~nm}$ with a binning factor of 2. Due to the limitation of the X-ray source, the total scanning time was about $60 \mathrm{~s}$. The image acquisition system was enclosed in a light-tight environment to avoid the outside light effect.

In the XLT reconstruction, the mouse was discretized into 31189 tetrahedral-elements and 6341 nodes, from the micro-CT results by AMIRA. We compared our method with traditional XLT $l_{1}$ regularization method [2] to validate the effectiveness of our proposed method. In the comparison, two benchmarks were applied to evaluate the reconstruction results, including location error and the dice coefficient [21]. The homogeneous absorption coefficient and reduced scattering coefficient were used in the reconstruction processes for three corresponding wavelengths [22]. For the microCT information, the reconstruction was performed using
TABLE 2: Reconstruction result of the two methods.

\begin{tabular}{lcc}
\hline & Location error $(\mathrm{mm})$ & Dice coefficient \\
\hline Proposed method & $1.1 \mathrm{~mm}$ & 0.4 \\
Traditional XLT method & $1.8 \mathrm{~mm}$ & 0.1 \\
\hline
\end{tabular}

the filtered back projection (FBP) method [23]. From the measured data, the distribution of the luminescent material could be reconstructed by the above mentioned method. The center of the capillary in the mouse was $21.2,21.5$, or 13 $\mathrm{mm}$ and was obtained from the micro-CT reconstruction result. Figure 3(a) shows the experimental surface data of the mouse. The color bar in Figure 3(a) shows the gray level of the image, whose unit is ADU (Analog to Digital Unit) and is ignored. Figure 3(b) shows the sectional view of the results with proposed method while Figure 3(c) shows the sectional view of the results with traditional method in which the black circle shows the actual material source. Figure 3(b) shows that the proposed method can better reveal the contour of the actual source than the traditional one. From Table 2, it can be seen that the proposed method can achieve smaller location error and larger dice coefficient than the traditional one, which means that the reconstruction results are more similar to the actual source in the proposed method. Both the location error and the dice coefficient in the results can prove that the proposed method can alleviate the ill-posedness and achieve good imaging quality. However, the light yield $\varepsilon$ of the material could not be obtained in our present system conditions. Hence, the quantity reconstruction results were not discussed and will be studied in future. Therefore, the unit of Figures 3(b) and 3(c) was ignored.

\section{Discussion}

Based on the above experiments, we have demonstrated that the proposed cone beam X-ray luminescence tomography imaging based on KA-FEM method is available to in vivo small animal imaging. The comparison between the proposed method and the traditional one shows that the proposed method can alleviate the ill-posedness and achieve good imaging quality from both the location error and the dice 


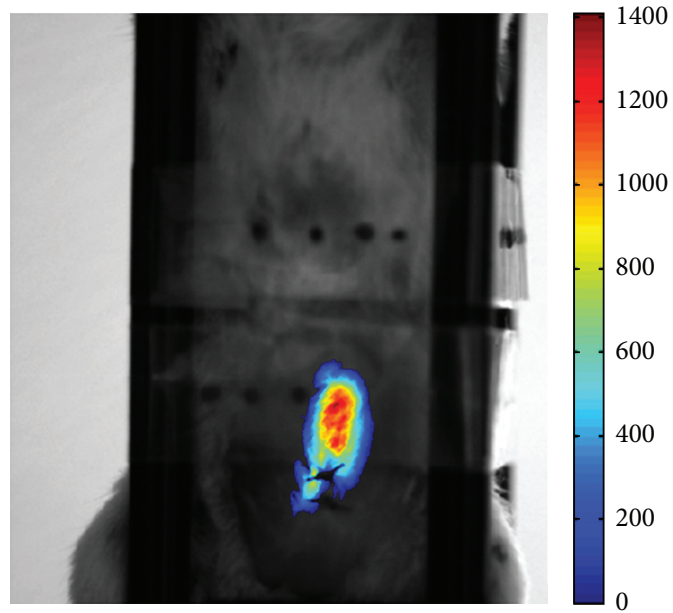

(a)

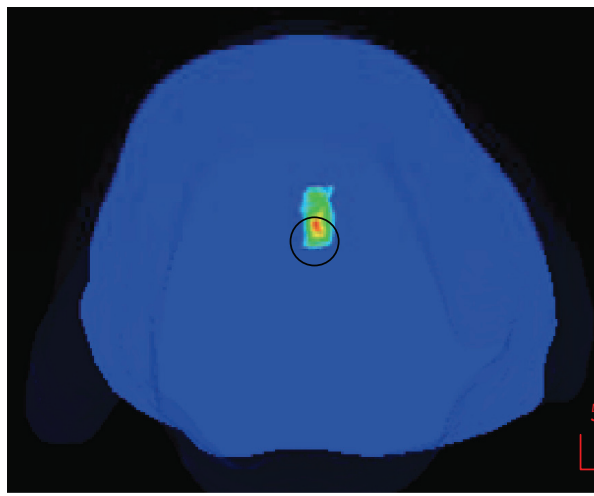

(b)

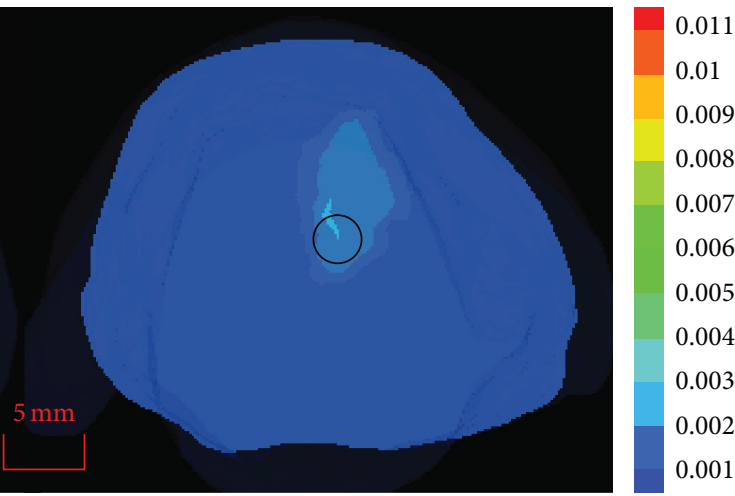

(c)

FIGURE 3: (a) Optical image of in vivo small mouse; (b) sectional view of the results with proposed method; (c) sectional view of the results with traditional method.

coefficient. Even though the spatial resolution of the reconstruction is relatively lower than that of the narrow beam XLT [1], the scanning time in our method could be shortened within $60 \mathrm{~s}$ with four angle scanning procedures while the reconstruction can be improved by multispectral imaging. However, the time resolution can be improved if the distance between the material and the X-ray source become smaller with larger FOV of the micro-CT system. The time resolution can also be improved by using a larger voltage or current of the X-ray source within less than $30 \mathrm{~s}$.

The method applied in [13] has also applied multiwavelength information and multilevel mesh strategy in the reconstruction. However, the structural information has been used as important information to realize the quantitative analysis, so the reconstruction error in [13] is smaller than the proposed one. If the reconstruction is conducted without the structural information, the FEM-FEM can achieve the location error of $1 \mathrm{~mm}$ and the dice coefficient of 0.38 , which is at the same level with the proposed one. Hence, both of them can improve the quality of XLT imaging. However, the KA module in our proposed method can realize reconstruction directly with CT voxel data. This can avoid the tedious segmentation and gridding work in traditional XLT imaging and improve the efficiency of the data process and analysis.
Due to the limitation of the personal computer, we cannot deal with the over-large number of the CT voxel data and realize the reconstruction, even though it may be solved in the future with the development of computer science. As a novel imaging technology, the imaging depth of XLT is limited by the material, system, and reconstruction method. It can be improved by increasing optical signal excited by increasing the tube voltage and current of X-ray source, using larger content of material with higher light yield. The influence of these related aspects is a significant task for our further study. Meanwhile, we apply homogeneous model in reconstruction with multispectral data in in vivo experiment. It can be deduced from Figure 2 that heterogeneous model for XLT reconstruction may be reasonable because of the difference in optical parameters of different tissues. Unfortunately, in our in vivo experiment, a plastic capillary filled with the $\mathrm{Gd}_{2} \mathrm{O}_{2} \mathrm{~S}$ : Tb nanophosphor was implanted into abdomen of the mouse, which may cause large artifacts in CT image and make it difficult to realize the accurate tissue segmentation. With an imprecise tissue segmentation, the reconstruction error of heterogeneous model is much larger than that of homogeneous model. The limitation of tissue data and tissue segmentation cannot fully support the heterogeneous reconstruction in our XLT experiment. With improvement 
of the segmentation technique and experiment conditions, we will further conduct the heterogeneous reconstruction to study the tissue difference in XLT imaging.

\section{Competing Interests}

The authors declare that there is no conflict of interests regarding the publication of this paper.

\section{Acknowledgments}

This work was supported by the National Natural Science Foundation of China (NSFC) under Grant nos. 61601154, 61601363, 81227901, and 61405149, the Scientific Research Foundation of Northwest University under Grant nos. 338020020 and 338050042, the Scientific Research Foundation of Hangzhou Dianzi University under Grant no. ZX160203302002-003, and the Beijing Municipal Natural Science Foundation under Grant no. 7142012.

\section{References}

[1] G. Pratx, C. M. Carpenter, C. Sun, R. P. Rao, and L. Xing, "Tomographic molecular imaging of X-ray-excitable nanoparticles," Optics Letters, vol. 35, no. 20, pp. 3345-3347, 2010.

[2] D. Chen, S. Zhu, H. Yi et al., "Cone beam X-ray luminescence computed tomography: a feasibility study," Medical Physics, vol. 40, no. 3, Article ID 031111, 2013.

[3] X. Chen, J. Liang, X. Cao et al., "Feasibility study of endoscopic X-ray luminescence computed tomography: simulation demonstration and phantom application," Journal of Applied Physics, vol. 114, no. 8, Article ID 084701, 2013.

[4] H. Chen, T. Moore, B. Qi et al., "Monitoring pH-triggered drug release from radioluminescent nanocapsules with X-ray excited optical luminescence," ACS Nano, vol. 7, no. 2, pp. 1178-1187, 2013.

[5] X. Liu, Q. Liao, and H. Wang, "In vivo X-ray luminescence tomographic imaging with single-view data," Optics Letters, vol. 38, no. 22, pp. 4530-4533, 2013.

[6] F. Leblond, S. C. Davis, P. A. Valdés, and B. W. Pogue, "Preclinical whole-body fluorescence imaging: review of instruments, methods and applications," Journal of Photochemistry and Photobiology B: Biology, vol. 98, no. 1, pp. 77-94, 2010.

[7] C. M. Carpenter, G. Pratx, C. Sun, and L. Xing, "Limited-angle $\mathrm{X}$-ray luminescence tomography: methodology and feasibility study," Physics in Medicine and Biology, vol. 56, no. 12, pp. 34873502, 2011.

[8] C. T. Badea, I. N. Stanton, S. M. Johnston, G. A. Johnson, and M. J. Therien, "Investigations on X-ray luminescence CT for small animal imaging," Proceedings of SPIE-The International Society for Optical Engineering, vol. 8313, Article ID 83130T, 2012.

[9] G. Wang, Y. Li, and M. Jiang, "Uniqueness theorems in bioluminescence tomography," Medical Physics, vol. 31, no. 8, pp. 22892299, 2004.

[10] C. Kuo, H. Ahsan, J. Hunter et al., "In vivo bioluminescence tomography using multi-spectral and multiple-perspective image data," Biomedical Optics, Technical Digest (CD) Paper TuG4, Optical Society of America, 2006.

[11] C. Kuo, O. Coquoz, T. L. Troy, H. Xu, and B. W. Rice, "Threedimensional reconstruction of in vivo bioluminescent sources based on multispectral imaging," Journal of Biomedical Optics, vol. 12, no. 2, Article ID 024007, 2007.
[12] Y. Tian, W.-H. Cao, X.-X. Luo, and Y. Fu, "Preparation and luminescence property of $\mathrm{Gd}_{2} \mathrm{O}_{2} \mathrm{~S}$ : Tb X-ray nano-phosphors using the complex precipitation method," Journal of Alloys and Compounds, vol. 433, no. 1-2, pp. 313-317, 2007.

[13] D. Chen, S. Zhu, X. Chen et al., "Quantitative cone beam $\mathrm{X}$-ray luminescence tomography/X-ray computed tomography imaging," Applied Physics Letters, vol. 105, no. 19, Article ID 191104, 2014.

[14] X. Wang, X. Cao, B. Zhang, F. Liu, J. Luo, and J. Bai, "A hybrid reconstruction algorithm for fluorescence tomography using Kirchhoff approximation and finite element method," Medical and Biological Engineering and Computing, vol. 51, no. 1-2, pp. 7-17, 2013.

[15] A. J. Chaudhari, F. Darvas, J. R. Bading et al., "Hyperspectral and multispectral bioluminescence optical tomography for small animal imaging," Physics in Medicine and Biology, vol. 50, no. 23, pp. 5421-5441, 2005.

[16] H. Dehghani, S. C. Davis, S. Jiang, B. W. Pogue, K. D. Paulsen, and M. S. Patterson, "Spectrally resolved bioluminescence optical tomography," Optics Letters, vol. 31, no. 3, pp. 365-367, 2006.

[17] J. Ripoll, V. Ntziachristos, R. Carminati, and M. Nieto-Vesperinas, "Kirchhoff approximation for diffusive waves," Physical Review E, vol. 64, no. 5, Article ID 051917, 2001.

[18] M. S. Patterson, B. Chance, and B. C. Wilson, “Time resolved reflectance and transmittance for the noninvasive measurement of tissue optical properties," Applied Optics, vol. 28, no. 12, pp. 2331-2336, 1989.

[19] R. Aronson, "Boundary conditions for diffusion of light," Journal of the Optical Society of America A, vol. 12, no. 11, pp. 2532 2539, 1995.

[20] W. Sun and Y.-X. Yuan, Optimization Theory and Methods, Springer, New York, NY, USA, 2006.

[21] D. Chen, S. Zhu, X. Cao, F. Zhao, and J. Liang, "X-ray luminescence computed tomography imaging based on X-ray distribution model and adaptively split bregman method," Biomedical Optics Express, vol. 6, no. 7, pp. 2649-2663, 2015.

[22] Y. Lv, J. Tian, W. Cong et al., "Spectrally resolved bioluminescence tomography with adaptive finite element analysis: methodology and simulation," Physics in Medicine and Biology, vol. 52, no. 15, pp. 4497-4512, 2007.

[23] J. Hsien, Computed Tomography Principles, Design, Artifacts, SPIE Press, 2003. 

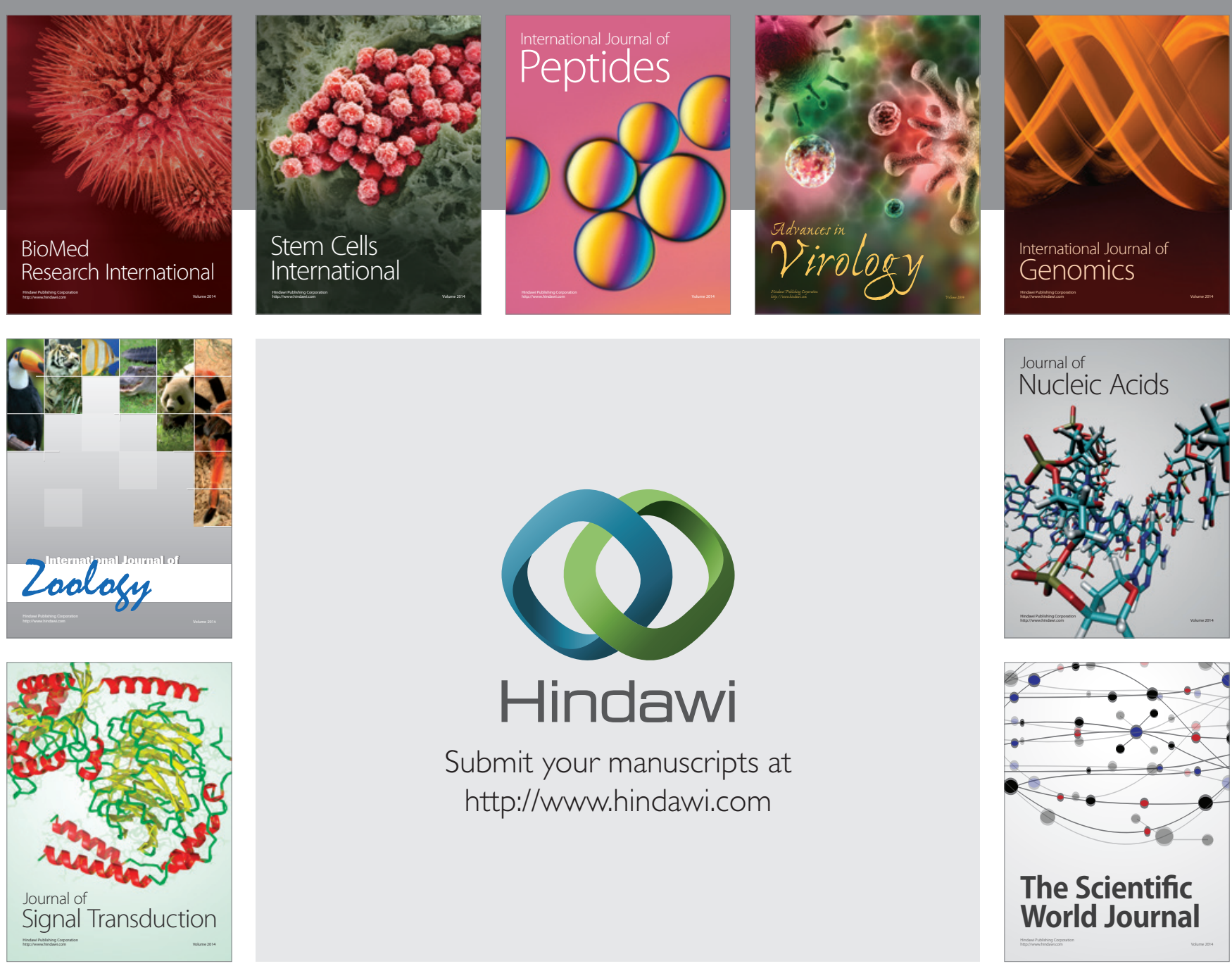

Submit your manuscripts at

http://www.hindawi.com
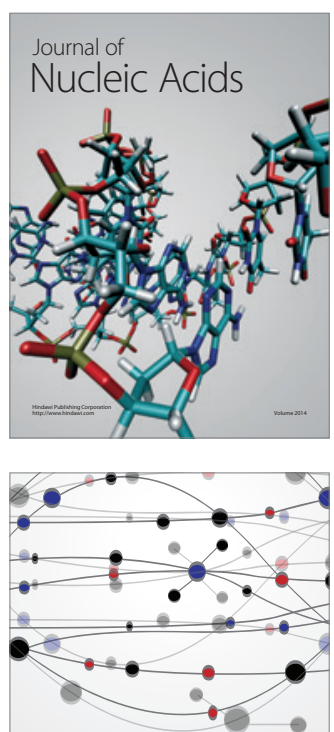

The Scientific World Journal
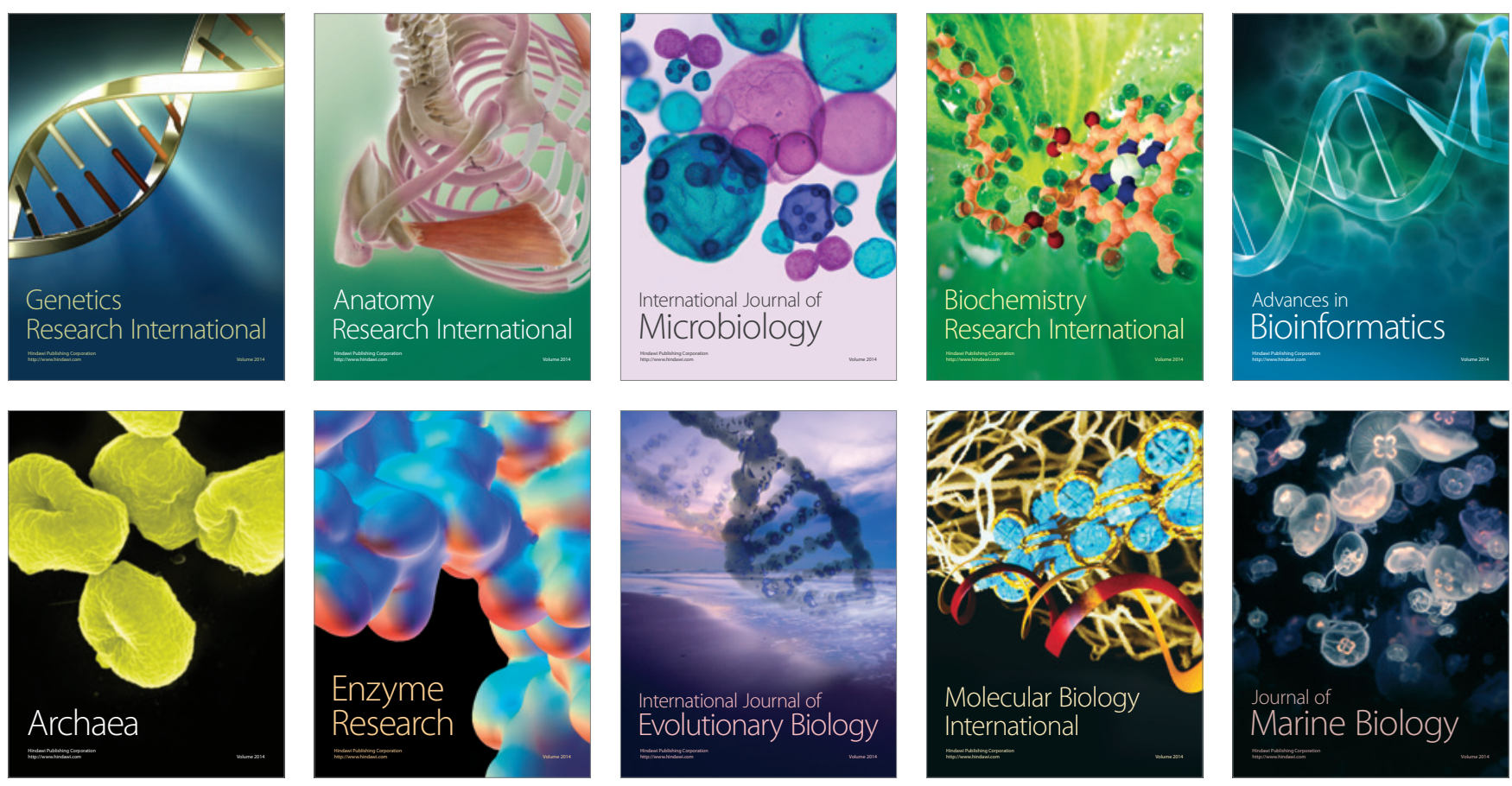